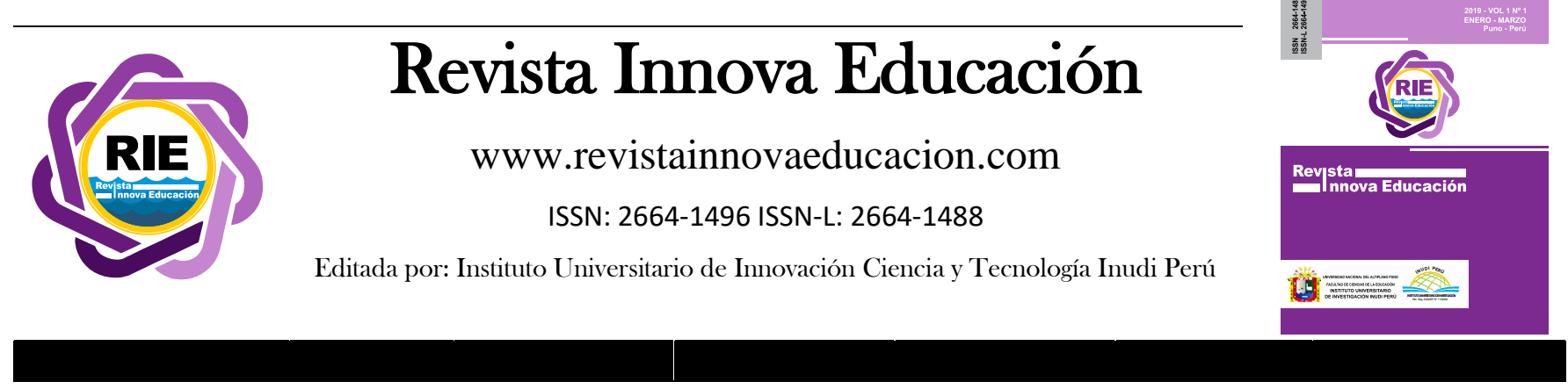

\title{
Efecto de la educación sobre la pobreza monetaria en las regiones del Perú
}

\author{
Luis Arias ${ }^{1}$; Henry Sucari² \\ DOI: https://doi.org/10.35622/j.rie.2019.01.009
}

Effect of education on monetary poverty in the regions of Peru

${ }^{1}$ Investigador independiente de la región Puno; Email: feroresa01@gmail.com
${ }^{2}$ Pontificia Universidad Católica del Perú; Email: henry.sucari@pucp.edu.pe

Recibido el 15/01/2019/ Aceptado el 27/01/2019

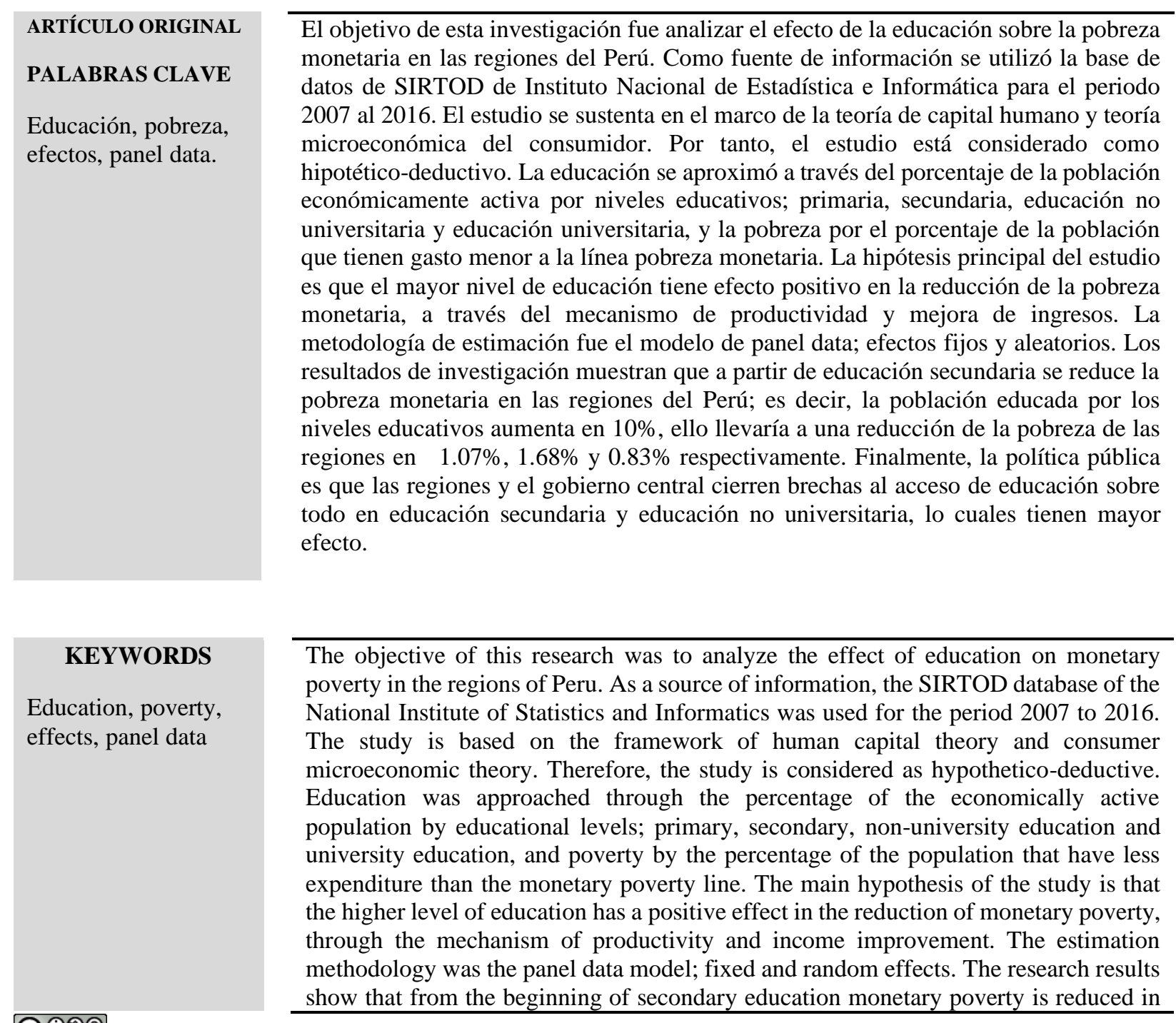


the regions of Peru; that is, the population educated by educational levels increases by $10 \%$, this would lead to a reduction of poverty in the regions by $1.07 \%, 1.68 \%$ and $0.83 \%$ respectively. Finally, public policy is that the regions and the central government close gaps in access to education, especially in secondary education and non-university education, which have the greatest effect.

\section{INTRODUCCIÓN}

En la última década ${ }^{1}$, la inversión en educación se ha incrementado sustancialmente; el promedio mundial de gasto en educación como porcentaje PBI alcanzó de $4.78 \%$ para el 2014 (Banco Mundial, 2014). El desempeño peruano en el contexto mundial, estuvo por debajo de esta cifra y por debajo de América Latina, sin embargo, no fue la peor. Si comparamos este indicador con respecto a los países de Sudamérica, entonces fue la región con menor inversión. De hecho, a nivel de Sudamérica, Bolivia es el país que más invierte en educación con 7.28\% del PBI (2014), seguida de Brasil 5.99\% (2013), Argentina 5.33\% (2014), Ecuador 5.24\% (2014), Chile 4.92\% (2015) y demás.

Sin embargo, a pesar del incremento de gasto en educación peruana durante la última década; de 2.7\% del 2008 a 3.9\% al 2017 como porcentaje del PBI (MINEDU, 2017), Perú sigue siendo la región que menos invierte en educación. Sumando a ello, la heterogeneidad de inversión entre las regiones.

La baja inversión en educación peruana estuvo acompañado con el aumento de mayores logros educativos (MINEDU, 2017); la tasa de analfabetismo (población de 15 años a más) se redujo de $8.2 \%$ a $5.8 \%$, mejorando también la calidad educativa. Asimismo, la tendencia vino acompañado con la reducción de la pobreza monetaria; este se redujo de $37.3 \%$ a $21,9 \%$ en la última década (INEI, 2017).

De acuerdo a la literatura económica, la mayor inversión en educación lleva incrementar el stock de capital humano; es decir a mayor gasto en educación, mayor logro educativo. Y este, lleva al crecimiento del producto per cápita, las cuales se traducen en mayor consumo de los hogares y finalmente este tiene efecto positivo en la reducción de la pobreza monetaria (Sala-i-Martin, 1995 citado en Pereyra, 2002). Además, el gasto en educación está dirigido a reducir el atraso económico, incrementar la productividad del trabajo, mejorar la distribución del ingreso y la reducción de la pobreza.

En este contexto, la investigación tiene como objetivo analizar el efecto de la educación sobre la pobreza monetaria en las regiones del Perú para el periodo 2007 - 2016. La variable de educación se aproximó a través del porcentaje de la Población Económicamente Actividad (población en edad trabajar) por niveles educativos: PEA con educación primaria, PEA con educación secundaria, PEA con educación no universitaria y PEA con educación universitaria. En cambio, la variable de pobreza por el porcentaje de la población que tienen gasto de consumo menor a la línea pobreza monetaria. Para medir adecuadamente el efecto de la educación sobre la pobreza se ha controlado por variables de la estructura económica de las regiones (variable proxy PBI) y otras del sector educación. En todos los casos la información proviene de Sistema de Información Regional para Toma de Decisiones - SIRTOD del Instituto Nacional de Estadística e Informática (INEI).

El marco teórico que se sustenta el estudio es la teoría de capital humano y la teoría

${ }^{1} 2007$ al 2016 
microeconómica de consumidor. Por tanto, esta investigación está considerado como método de investigación hipotético-deductivo (Mendoza, 2014). La metodología de estimación empleada fue el modelo de panel data; efectos fijos y aleatorios, ya que los datos son de sección cruzada.

La literatura respecto al tema es diversa. El principal autor guía del estudio fue Mendoza (2003), quien analiza la relación entre la educación y el problema de la pobreza departamental formulando un modelo teórico de corte microeconómico del consumidor. Los resultados que llega el autor es que, la PEA con educación superior y la tasa de retiros en la educación secundaria son las dos principales variables educativas que determinan el nivel de la pobreza departamental en el Perú. En cambio Aguado et. al (2006), en su investigación titulada "Una aproximación empírica a la relación entre educación y pobreza", quien estudia mediante el modelo logit de elección múltiple ordenado y modelos de ecuaciones estructurales, concluye que existe relación negativa entre la educación y la pobreza; es decir mayor educación, menor es la pobreza. Asimismo, indica que la inversión en capital humano mediante la educación es un factor clave para mejorar el ingreso de las personas, ésta por sí sola no es suficiente para alterar el cambio de condición de pobre a no pobre. Por su parte Olivia (2017) en su estudio relación entre el nivel educativo y la pobreza en el Perú, aproximando la educación como años promedio de escolaridad de la población de 25 a 60 años de edad y la pobreza por línea de pobreza, llega al siguiente resultado: que la mayor educación reduce la pobreza en las regiones. Y finalmente Gonzales (2017), llega a los resultados mismo resultados señalados. En términos de magnitudes y coeficientes los resultados de estudios de los autores señaladas se muestran en la parte de discusión.

\section{MÉTODOS Y MATERIALES}

\section{Marco teórico sobre la relación educación y la pobreza}

El presente estudios sigue de cerca el marco teórico de capital humano, principalmente en el estudio empírico de Rodríguez (2017). La tesis fundamental de que el capital humano explica una parte del crecimiento económico, se puede aproximar mediante el modelo básico, de Lucas (1998) (citado en Rodríguez, 2017):

Dónde:

$$
Y_{t}=K_{t}^{\alpha}\left(u h_{t} L_{b t}\right)^{1-\alpha} H_{t}^{\gamma}
$$

$\boldsymbol{Y} \quad$ : Producto total de economía del país

$\boldsymbol{K} \quad$ : Capital físico

$\boldsymbol{L}_{\boldsymbol{b}} \quad$ : Número de trabajadores en el sector productor de bienes y servicios no educativos.
$\boldsymbol{h}$ : Capital humano dentro de la empresa
$\boldsymbol{H}$ : Capital humano de la economía
$\boldsymbol{u} \quad$ : Porcentaje del tiempo dedicado al trabajo en relación al tiempo total para estudiar y trabajar
$\boldsymbol{\alpha} \quad$ : Está entre 0 y 1
$\gamma \geq 0$ : Parámetro de acervo en el capital humano

Siguiendo las ecuaciones propuestas por el autor y siguiendo adicionalmente el estudio de Barro \& Sala i Martin (2009), la inversión en educación tiene efecto positivo en aumentar el stock de capital humano, y este a su vez el producto per cápita. Si incorporamos a ello las familias que 
maximiza el bienestar a través del consumo, entonces el producto per cápita se traduce en el consumo per cápita $\left(C_{t}=Y_{t}\right)$. Por tanto, a través de las ecuaciones de Romer (2006) el consumo depende del producto per cápita, y este es explicado por el stock de capital humano, lo que se traduce en mayor consumo, y finalmente en la reducción de la pobreza (véase Figura 1).

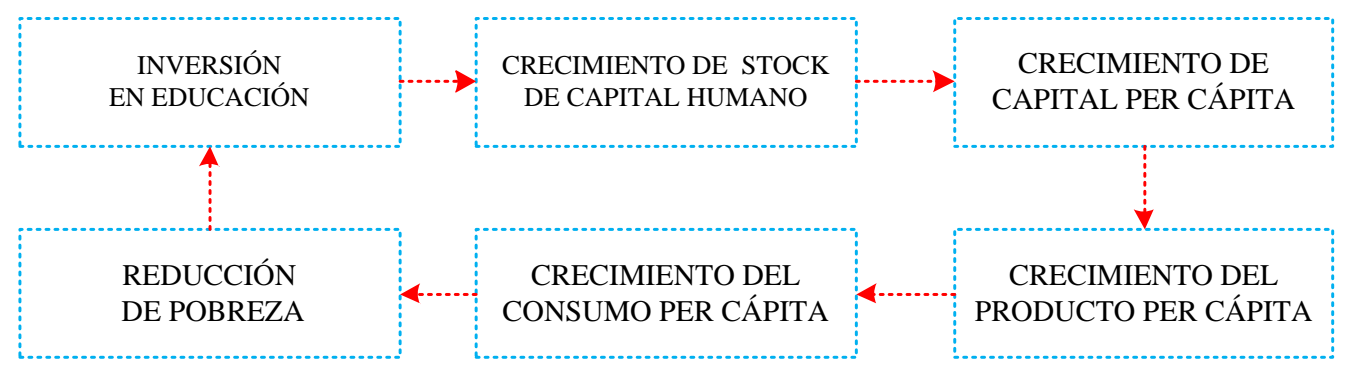

Figura 1. Mecanismo de transmisión de la educación sobre la pobreza monetaria, nivel macroeconómico Fuente: Adaptada al estudio de Rodríguez (2017)

Desde la perspectiva microeconómica la relación entre la pobreza y la educación se puede partir de una función de utilidad ${ }^{2}$, donde el individuo máximo su utilidad consumiendo bienes y servicios y este está sujeta a su restricción presupuestaria. En términos del autor la pobreza se puede determinar a través del consumo o gasto del individuo:

"Dado los precios (P), se puede definir la situación de un individuo como pobre o no pobre en función a que su nivel de consumo esté por debajo o por encima de un nivel de consumo mínimo (CM); tal consumo mínimo es una especie de una "línea de consumo" Así, un individuo es considerado como pobre cuando tiene niveles de consumo menores que la mencionada línea de consumo, en caso contrario, es considerado como no pobre" (Mendoza, 2003, p 08).

La Figura 2 muestra la modelo adaptada al autor, según el modelo microeconómico la educación tiene efecto inmediato en la productividad del trabajo; es decir, personas más educadas son más productivos, posteriormente este eleva el ingreso o el salario. Por tanto, el individuo puede consumir más de bienes y servicios, con ello saldría de la línea de la pobreza monetaria.

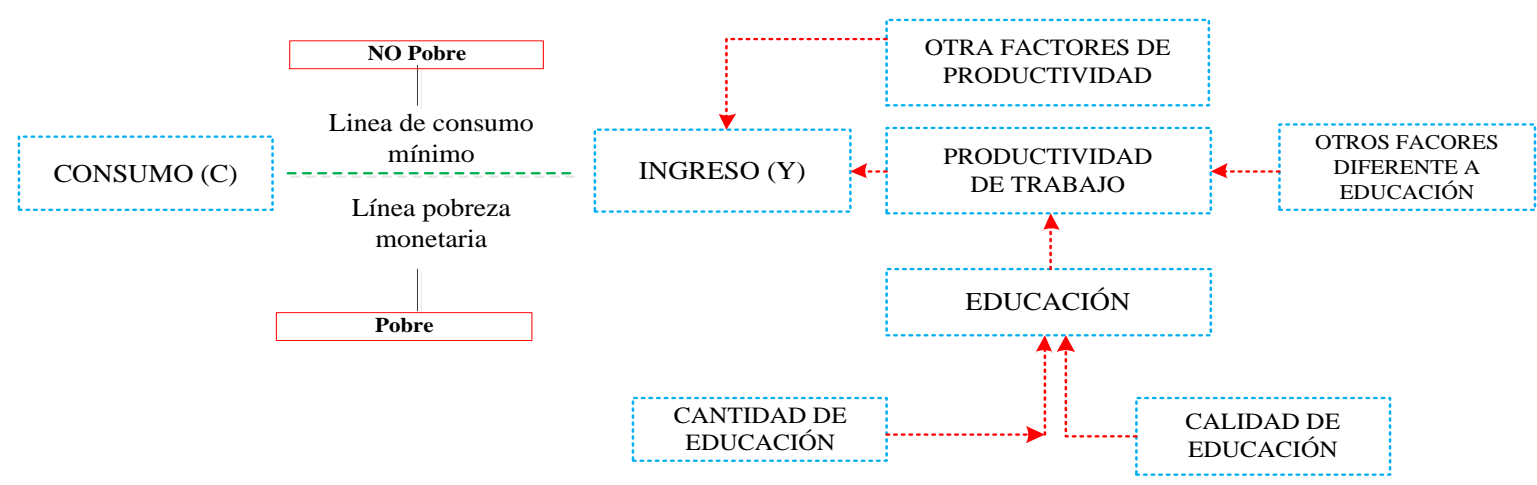

Figura 2. Mecanismo de transmisión de la educación sobre la pobreza monetaria, nivel microeconómico Fuente: Adaptada al estudio de León (2003)

\section{Método de investigación}

${ }^{2}$ Para más información del tema se sugiere ver Mendoza (2003). 
El tipo de investigación es no experimental, y su forma es el diseño transeccional y longitudinal. El método de investigación hipotético-deductivo (Mendoza, 2014), ya que la hipótesis del trabajo proviene del marco teórico, por tanto, es considerado como "investigación básica" o "metodología de investigación científica".

\section{Población y muestra}

La población del presente estudio está conformada por las regiones del Perú (24 regiones), y la muestra representa el universo del estudio en el periodo 2007 y 2016 . En todos los casos la información proviene de Sistema de Información Regional para Toma de Decisiones - SIRTOD del Instituto Nacional de Estadística e Informática (INEI).

\section{Modelo econométrico}

El modelo econométrico para contrastar la hipótesis entre la educación y la pobreza se sustenta en la teoría microeconómica de Mendoza (2003), siendo la ecuación teórica:

Dónde:

$$
I P=f(P E A P R I M, P E A S E C, P E A N O U N I, P E A U N I, X)
$$

IP

PEAPRIM

PEASEC

PEANOUNI

PEAUNI

$\mathrm{X}$
: Índice de pobreza regional

: Porcentaje de PEA con educación primaria o menos

: Porcentaje de PEA con educación secundaria

: Porcentaje de PEA con educación superior no universitaria

: Porcentaje de PEA con educación superior universitaria

: Otras variables que pueden tener efecto

La ecuación econométrica a regresiones es la siguiente:

$$
\begin{gathered}
\text { IP }_{i t}=\beta_{0}+\beta_{1} P E A P R I M_{i t}+\beta_{2} P_{E A S E C}+\beta_{3} P E A N O U N I_{i t}+\beta_{4} P \text { PAUNI }_{i t}+\beta_{5} X_{i t} \\
+\alpha_{i}+\varepsilon_{i t}
\end{gathered}
$$

Donde $\beta_{0}$ es la constante del modelo, $\beta_{1}$ representa el impacto de PEA con educación primaria sobre la pobreza, de $\beta_{2}$ a $\beta_{4}$ representa los diferentes impactos de pasar una categoría de educación sobre la pobreza monetaria, $\alpha_{i}$ es el error que no cambia en el tiempo y $\varepsilon_{i t}$ es el error idiosincrático o error del modelo clásico, las cuales se distribuyen con media cero y varianza constante.

Siguiendo Wooldridge (2002) la técnica de estimación de estimación es el modelo panel data; efectos fijos y aleatorios, para ello, debe cumplir con todos los supuestos de la regresión clásica. El estudio se sustenta en programa estadístico STATA 13.

\section{RESULTADOS}


La Figura 03 muestra la educación aproximada a través del porcentaje de la Población Económicamente Activa (PEA) por niveles educativos; PEA con educación primaria o menos, PEA con educación secundaria, PEA con educación no universitaria y PEA con educación universitaria.

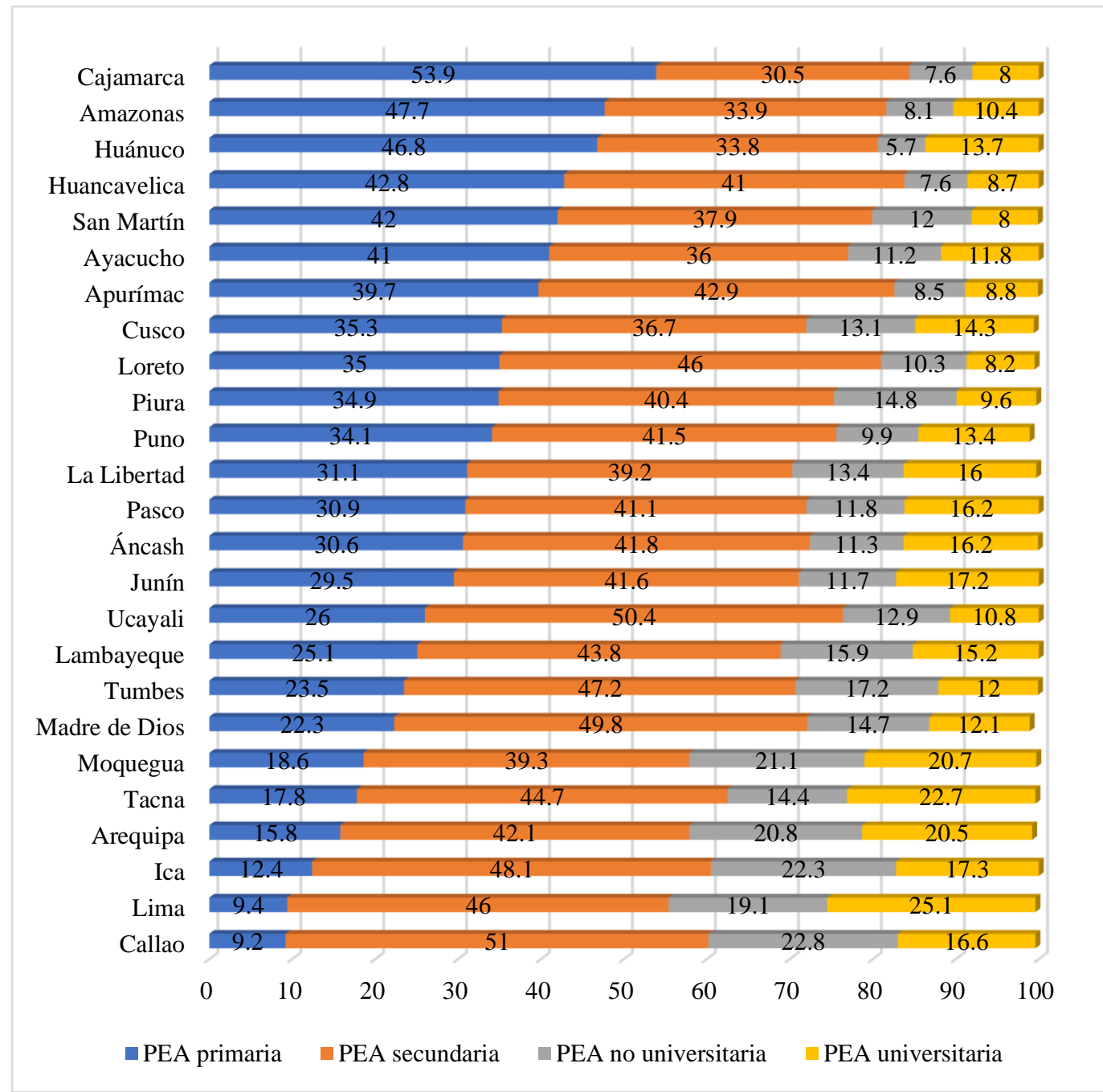

Figura 3. Población Económicamente Activa (PEA) según nivel educativo por regiones.

Fuente: INEI -2016

Cómo se puede observar las regiones Cajamarca, Amazonas, Huánuco, Huancavelica, San Martin, Ayacucho y Apurímac tienen alto nivel de PEA con educación primaria, por encima del 40\%. En tanto las regiones Callao, Ucayali, Madre de Dios, Ica, Tumbes, Lima, Loreto, Tacna y Lambayeque tienen más PEA con educación secundaria, por encima del 43\%. Por lado de PEA con educación superior no universitaria, aunque la cifra es baja, las regiones Callao, Ica, Moquegua, Arequipa, Lima, Tumbes, Lambayeque, Piura, Madre de Dios y Tacna lideran por encima del 14\%. Finalmente, las regiones Lima, Tacna, Moquegua, Arequipa, Ica, Junín, Callao, Pasco, Áncash, La Libertad y Lambayeque tiene la población más con PEA educada por encima del $15 \%$. De hecho, en la última década aumentaron el logro educativo y la PEA ocupada a partir de la educación secundaria, en cambio la PEA primaria se ha reducido.

Por su parte es importante considerar la transición de educación a diferentes niveles educativos. La Figura 04 muestra la tasa de transición a educación secundaria y superior por 
niveles de pobreza. Según el ESCALE (2016) a nivel nacional, el 97\% de estudiantes de nivel primaria siguen estudiando en el nivel secundario. En tanto, solo el $37 \%$ de estudiantes de nivel secundario continúan con los estudios superiores. A nivel de la pobreza y pobreza extrema la transición de primaria a secundaria en menor con respecto a los no pobres, y es más significativo en la extrema pobreza. Y la transición de secundaria a educación superior es más bajo aún. Generalmente en el pobre extremo la transición es solo del $15 \%$.

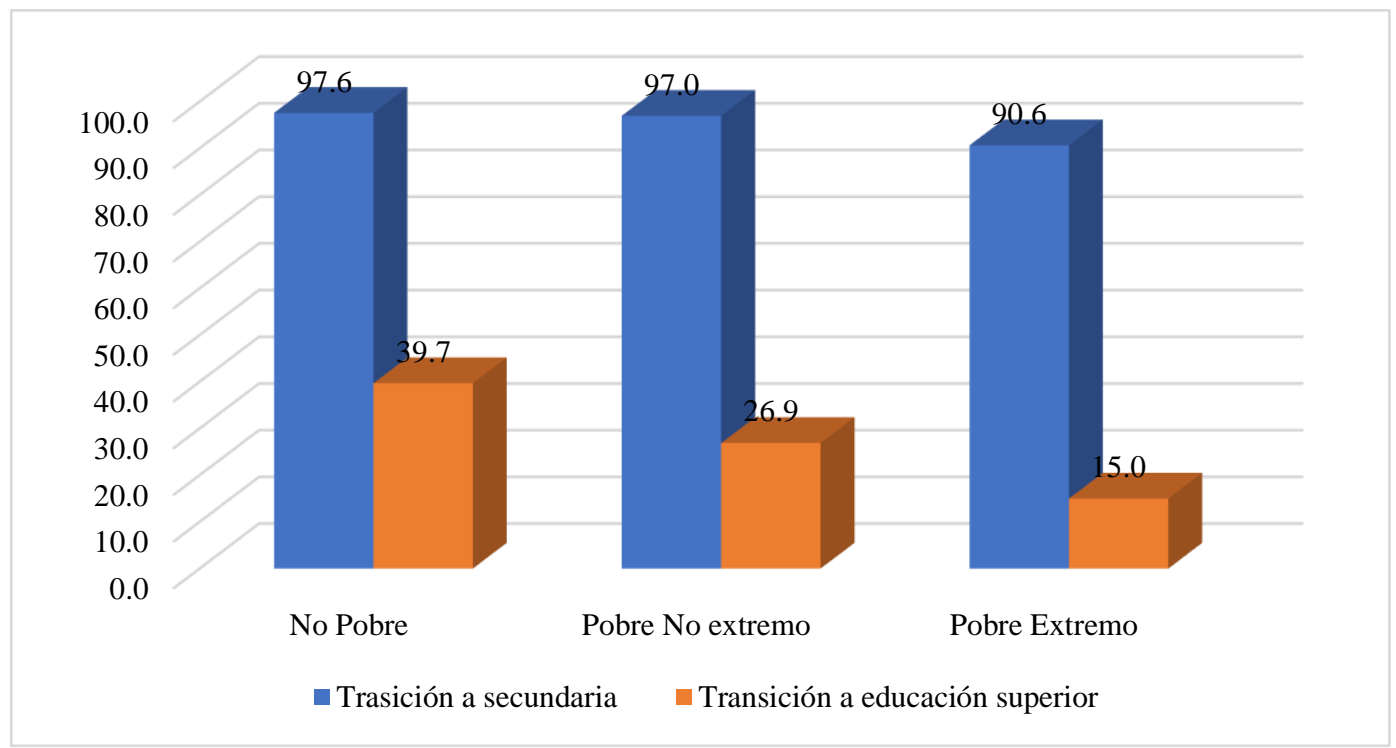

Figura 4. Tasa de transición a educación secundaria y superior por niveles de pobreza Fuente: ESCALE -2016

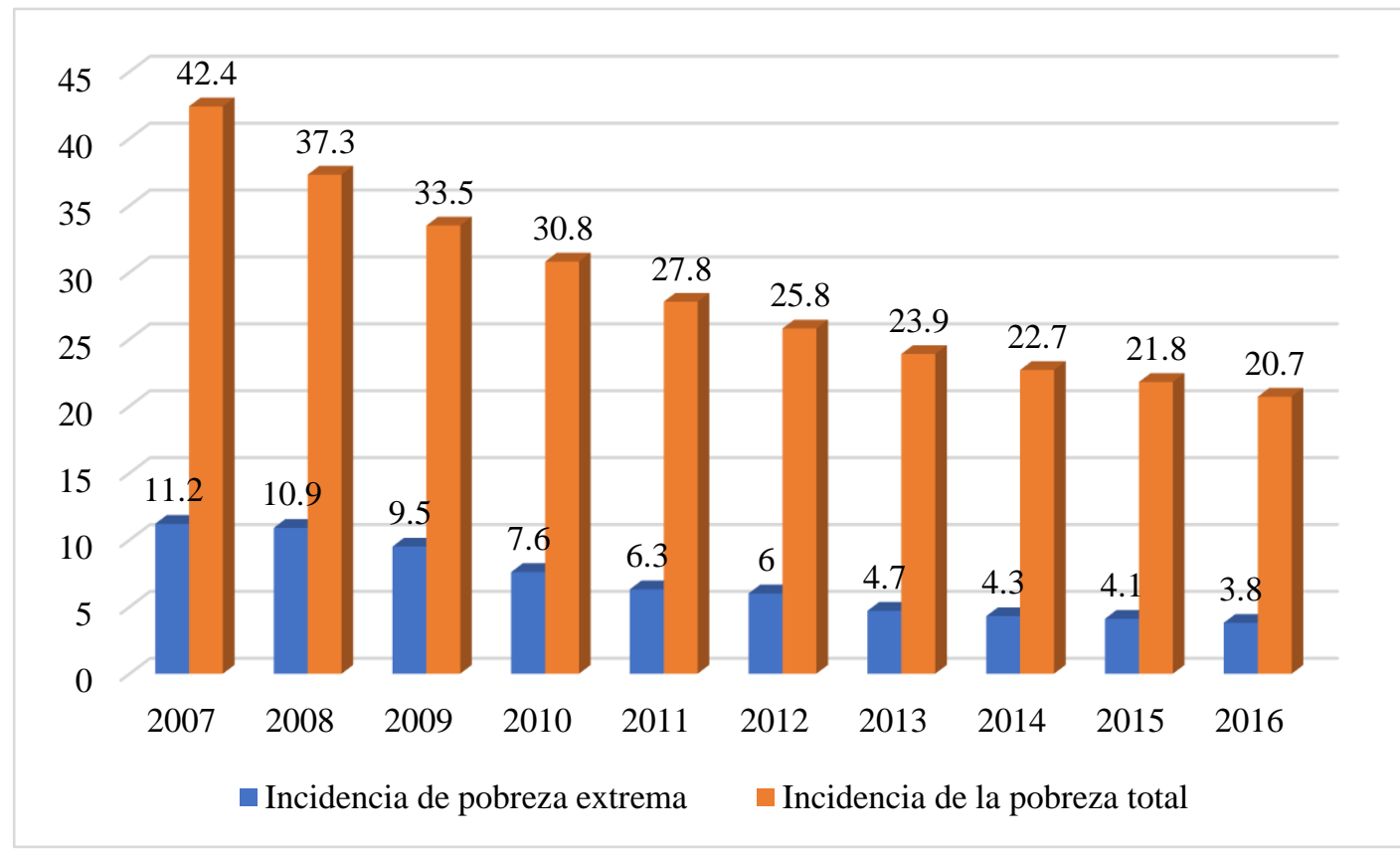

Figura 5. Evolución de la pobreza monetaria nacional, últimos 10 años Fuente: SIRTOD - INEI

Por lado de pobreza monetaria, se puede observar que durante la última década ha disminuido de $42.4 \%$ a $20.7 \%$, con una tasa de decrecimiento de $7.6 \%$ promedio anual (véase Figura 05). Cabe destacar que la pobreza se basa en la línea de la pobreza monetaria, este es asignado anualmente por el Instituto Nacional de Estadifica e Informática según al gasto de

\section{Luis Arias y Henry Sucari}


consumo de las familias. Por otro lado, se observa la pobreza extrema, este también ha disminuido en la última década de $11.2 \%$ a $3.8 \%$.

Del mismo modelo la cifra por regiones ha disminuido durante la última década, sin embargo, esta ha sido muy heterogénea. La Figura 06 muestra el mapa de pobreza monetaria a nivel de regiones del Perú. Las regiones con mayores niveles de pobreza son: Cajamarca, Ayacucho, Amazonas, Pasco, Huancavelica, Apurímac, Huánuco, Piura y Puno. Y las regiones con menores niveles de pobreza son: Madre de Dios, Ica, Moquegua, Arequipa, Tacna, provincia de Lima, y Ucayali.

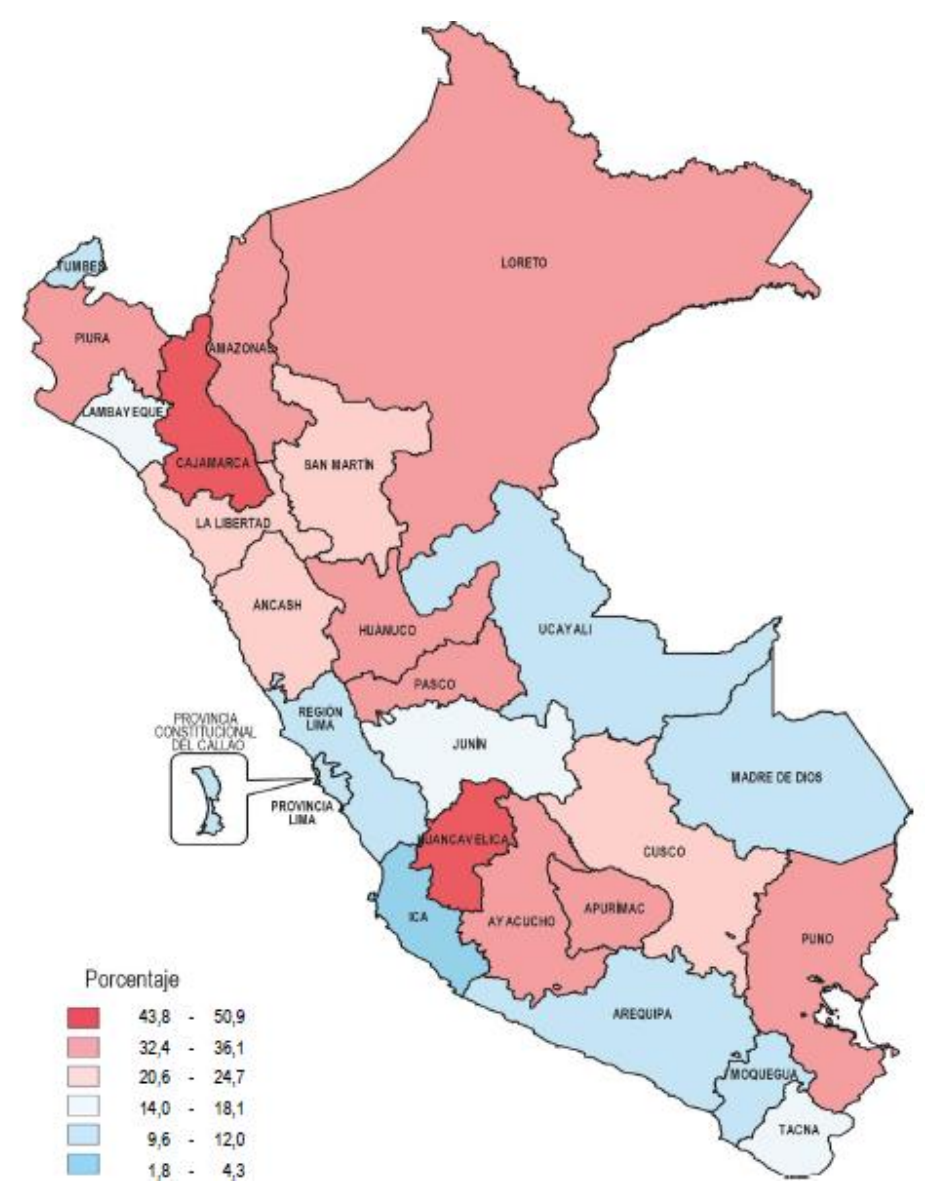

Figura 6: Evolución de la pobreza monetaria: Regiones del Perú

Fuente: INEI - 2016

Una vez descrita variables de educación y pobreza se procedió a realizar las correlaciones gráficas con el fin de determinar el efecto de la educación sobre la pobreza monetaria. La Figura 7 muestra la correlación entre la PEA con educación primaria y secundaria con la pobreza monetaria, donde se puede observar que, la primera muestra una relación positiva y la segunda negativa. En el primer caso se puede deducir que las regiones con mayor PEA en educación primaria suelen tener mayor pobreza, tales son las regiones Huancavelica, Cajamarca, Amazonas, Huánuco, Apurímac y Ayacucho. Por tanto, la educación primaria no es suficiente para reducir la pobreza monetaria. En cambio, en el segundo caso, la PEA secundaria es capaz de reducir la pobreza monetaria, este indicador es más efectiva en las regiones Callo, Madre de Dios, Ica, Ucayali, Lima Metropolitana, Ica y Tacna. 

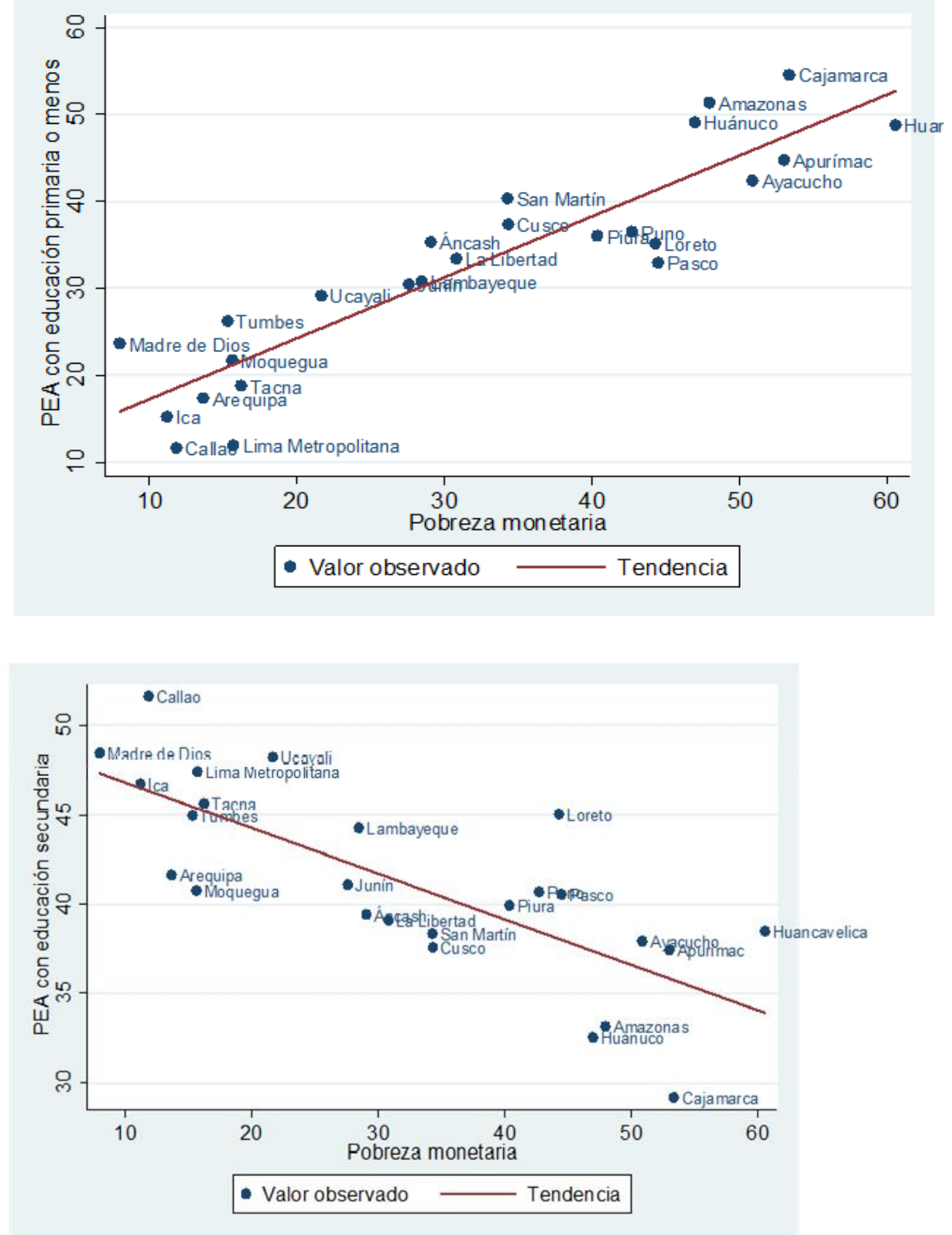

Figura 7: Relación entre PEA con educación primaria y secundaria sobre la pobreza monetaria. Fuente: Elaboración propia en base a datos de INEI

La educación superior representada por PEA con educación no universitaria y con educación universitaria, también tuvieron efecto positivo en la reducción de la pobreza monetaria, con mayor efectividad en las regiones de Costa (véase Figura 8). 

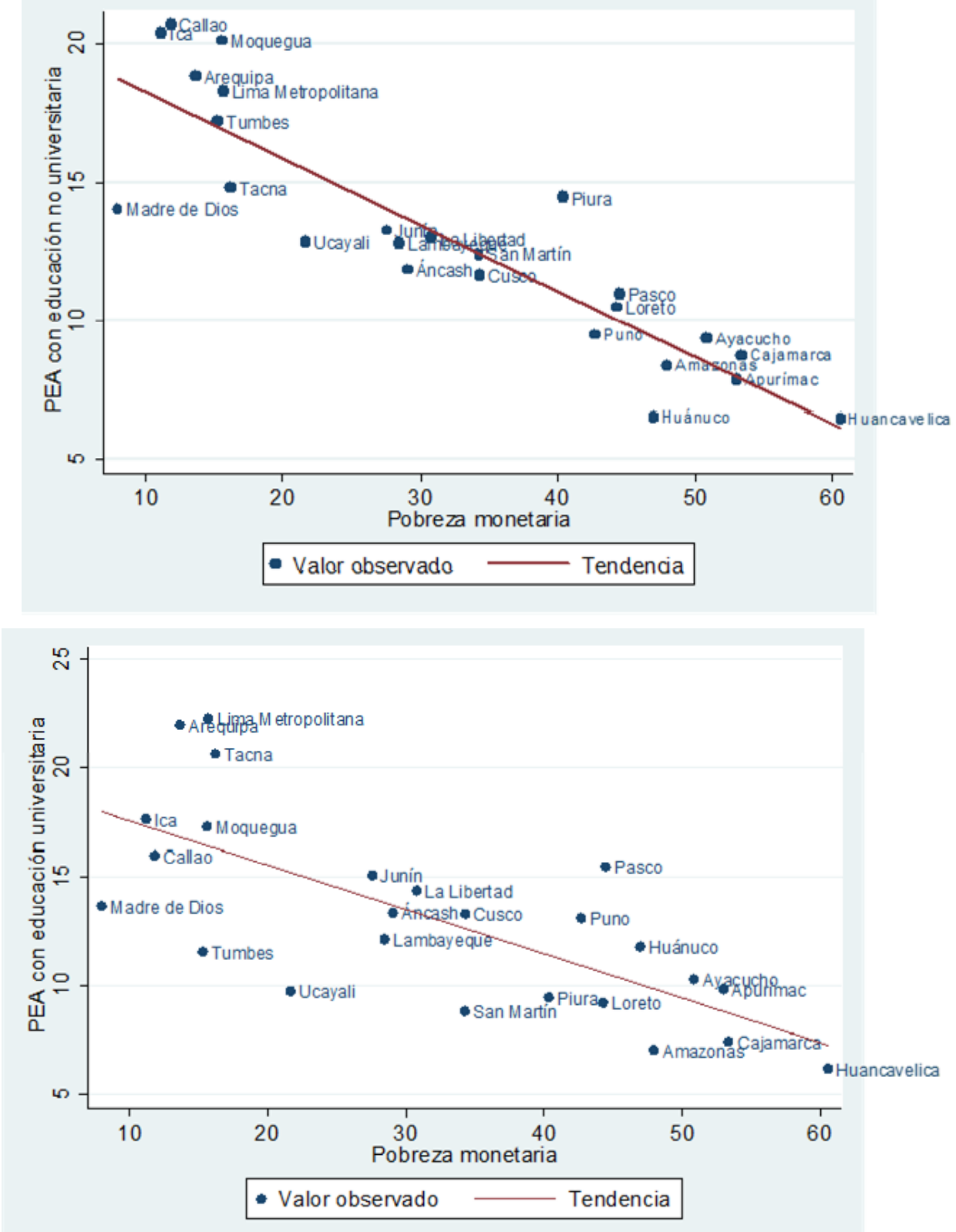

Figura 8: Relación entre PEA con educación primaria y secundaria sobre la pobreza monetaria. Fuente: Elaboración propia en base a datos de INEI

Una determinada la relación se estimó el efecto a través del modelo de panel data; efectos fijos y aleatorios, adicionalmente se estimó por el panel dinámico, siguiendo el modelo de Arellano \& Bond (1991) ello con fines de comparación. Para tal se cumplieron las pruebas de Breuch-Pagan y Hausman de exogeneidad del modelo, y en el caso de Arellano y Bond (1991), los supuestos de autocorrelación y el test de exogeneidad débil de Sargan ${ }^{3}$.

La Tabla 1 muestra los resultados de la estimación del modelo de efecto de educación sobre la pobreza de las regiones del Perú, como se puede observar en los tres modelos, la educación tiene una relación negativa con la pobreza monetaria, a excepción de la PEA con educación primaria, en todos los casos es significativa a 1\%, según $p$-valor. De la misma forma las variables de control muestran signos correctos de sus coeficientes y son significativas.

\footnotetext{
${ }^{3}$ Para ver la versión completa del estudio del método panel data: efectos fijos y aleatorios, y el Arellano y Bond (1991) escribir al correo del autor.
} 
Tabla 1 Resultados de estimación de la relación entre la educación y la pobreza monetaria

\begin{tabular}{|c|c|c|c|c|c|c|}
\hline \multirow[b]{4}{*}{ VARIABLES } & \multicolumn{6}{|c|}{ Variable dependiente: POBREZA MONETARIA } \\
\hline & \multicolumn{3}{|c|}{ POBREZA (I) } & \multicolumn{3}{|c|}{ POBREZA EXREMA (II) } \\
\hline & \multirow[b]{2}{*}{ FE } & \multicolumn{2}{|c|}{ XTABON } & \multicolumn{3}{|c|}{ XTABO } \\
\hline & & RE & $\mathrm{D}$ & FE & RE & ND \\
\hline \multirow[t]{3}{*}{ L.peaprim } & $0.2184 * * *$ & $0.2217 * * *$ & $0.2851 * * *$ & 0.0742 & $0.0995 * *$ & 0.0547 \\
\hline & & & & - & - & \\
\hline & - & - & - & $0.5965 * *$ & $0.6209 * *$ & \\
\hline peasec & $0.9630 * * *$ & $1.0722 * * *$ & $0.9998 * * *$ & $*$ & $*$ & -0.5678 \\
\hline
\end{tabular}

Educaci

ón

$\begin{array}{ccccccc} & - & - & - & 0.8463 * * & 0.9108 * * & \\ \text { peanouni } & 1.5722 * * * & 1.6814 * * * & 1.5701 * * * & * & * & -0.8220\end{array}$

\begin{tabular}{ccccccc} 
& - & - & - & $0.3616^{* *}$ & - \\
peauni & $1.0051 * * *$ & $0.8731 * * *$ & $0.9191 * * *$ & $*$ & $0.3097 * *$ & -0.2728 \\
\hline & & & & $0.2923 * *$ \\
L.lec & $0.8087 * * *$ & -0.1147 & $0.8729 * * *$ & $*$ & -0.0964 & 0.3084
\end{tabular}

Variable

s de

L.mate

$0.7000 * * * \quad-0.1443$

$0.7654 * * *$

$0.3242 * *-0.0555$

$-0.3412$

control

deser

$0.5663 * * *$

$-0.3842 * *$

$0.5725 * * *$

$-0.1836^{*}$

$-0.1137$

$-0.1621$

\begin{tabular}{ll} 
& lpbir \\
\hline Lezagose_int & L.pobrext_i \\
& $n t$
\end{tabular}

$-2.5550 *-4.6174 * * 2.9$

$9337 * * *$

$-0.5391 \quad-1.2510 \quad-0.6247$
$-0.0623$

\begin{tabular}{lcccccc}
\hline Constant & $\begin{array}{c}115.3456^{*} \\
* *\end{array}$ & $\begin{array}{c}168.6716^{*} \\
* *\end{array}$ & $\begin{array}{c}118.3299 * \\
* *\end{array}$ & $\begin{array}{c}50.3275^{*} \\
* *\end{array}$ & $\begin{array}{c}69.7564 * \\
* *\end{array}$ & 48.9639 \\
\hline $\begin{array}{l}\text { Observations } \\
\text { R-squared }\end{array}$ & 222 & 222 & 192 & 222 & 222 & 191 \\
$\quad$ within & 0.8286 & 0.7590 & & 0.6362 & 0.6012 & \\
$\quad$ between & 0.0439 & 0.8418 & & 0.0470 & 0.8535 & \\
$\quad$ overall & 0.5943 & 0.7434 & & 0.5383 & 0.6224 & \\
sigma_u & 9.7345 & 0.0000 & & 3.8334 & 0.0000 & \\
sigma_e & 6.9004 & 6.9004 & & 5.4027 & 5.4027 & \\
rho & 0.6656 & 0.0000 & & 0.3349 & 0.0000 & \\
Number of year & 10 & 10 & & 10 & 10 & 10 \\
\hline$* * * \mathrm{p}<0.01, * * \mathrm{p}<0.05, * \mathrm{p}<0.1$ & & & & &
\end{tabular}

FE: Método de efectos fijos

RE: Método de efectos aleatorios

XTABOND: Método de panel dinámico - Arellano y Bond (1991)

Fuente: Elaboración propia en base a resultados. 
Con fines de interpretación se basó en el modelo de efectos aleatorios. Según este modelo la PEA con educación primaria (peaprim) no llevaría a la reducción de la pobreza, todo lo contrario, llevaría a mayor pobreza. Puntualmente, un aumento del 10\% de PEA con educación primaria llevaría a un aumento de $0.22 \%$ de la pobreza. En tanto, la PEA con educación secundaria (peasec), con educación no universitaria (peanouni) y con educación universitaria (peauni), llevaría a la reducción de la pobreza monetaria. Si la cifra de las mismas aumentará en 10\%, esto se traduciría en una reducción de pobreza en $0.99 \%, 1.57 \%$ y $0.91 \%$ respectivamente. Como se puede apreciar la PEA con educación no universitaria tiene mayor efecto en la reducción de la pobreza, luego sigue la PEA con educación secundaria. Asimismo, la educación tiene efecto positivo en la reducción de la pobreza extrema, tal como se aprecia en la Tabla 1. Las variable de control como la estructura económica del distrito representado por el PBI (lpbi) y otras variables de educación tanto de calidad como cantidad educativa; logro suficiente en lectura (lec) y matemática (mate) y tasa de deserción en quinto año de secundaria (deser) mostraron correctos; es decir, que mayor PBI de las regiones tiene efecto positivo en la reducción de la pobreza, mayor logro educativo menores son los niveles de la pobreza y a mayor deserción en quinto grado de secundaria mayores serán la pobreza monetaria. Desde el punto de vista econométrico y estadístico, el R cuadrado (overall) es de 0.7434 indica que el modelo de pobreza es explicado en $74.34 \%$ por la variable de educación.

Las variable de control como la estructura económica del distrito representado por el PBI (lpbi) y otras variables de educación tanto de calidad como cantidad educativa; logro suficiente en lectura (lec) y matemática (mate) y tasa de deserción en quinto año de secundaria (deser) mostraron correctos; es decir, que mayor PBI de las regiones tiene efecto positivo en la reducción de la pobreza, mayor logro educativo menores son los niveles de la pobreza y a mayor deserción en quinto grado de secundaria mayores serán la pobreza monetaria.

\section{DISCUSIÓN}

Según los resultados de investigación se ha mostrado que la educación es efectiva para reducir la pobreza monetaria a partir de la educación secundaria, la educación primaria no es suficiente. Este último, según Mendoza (2003), estaría relacionado con los mecanismos de transmisión de educación a la pobreza, ya que el bajo nivel educativo tendrá en baja productividad del trabajo, y este en bajo nivel de ingresos y consumo de bienes y servicios, y por tanto mayor pobreza.

Comparando los resultados con otros estudios presentada en la evidencia empírica se ha llegado a resultados similares, con algunas excepciones. En caso de Mendoza (2003), la pobreza es reducida a partir de educación superior; es decir, un aumento en 1\% en la PEA con educación superior reduce en $0.72 \%$ el porcentaje de población departamental en estado de pobreza. En cambio Aguado et. al (2006), es más optimista ya que por cada $1 \%$ de escolaridad promedio de la población de 25 a 65 años, tendería a reducir la pobreza monetaria de las regiones en $13.11 \%$. Y finalmente Gonzales (2017), obtiene resultados similares al de Mendoza (2003).

Por lo anterior, la recomendación de política a las regiones o el gobierno central es aumentar la población PEA educada a partir de la educación secundaria y cierre de brechas en el nivel de educación superior universitaria y no universitaria.

\section{CONCLUSIÓN}


La educación tiene efecto positivo en reducción de la pobreza monetaria a niveles de regiones del Perú a partir de la educación secundaria. Los resultados muestran que la PEA con educación primaria no llevaría a la reducción de la pobreza, todo lo contrario, llevaría a mayor pobreza. En tanto, la PEA con educación secundaria, con educación no universitaria y con educación universitaria, llevaría a la reducción de la pobreza monetaria. Si la cifra la cifra de PEA con educación aumentara en $10 \%$ por niveles educativos (desde la educación secundaria), esto se traduciría en una reducción de pobreza monetaria en $0.99 \%, 1.57 \%$ y $0.91 \%$ respectivamente.

\section{REFERENCIAS BIBLIOGRÁFICAS}

Aguado, F., Girón, L., \& Salazar, F. (2006). Una aproximación empírica a la relacion entre la educación y pobreza. Problemas Del Desarrollo: Revista Latinoamericana de Economía, 38, 35-60.

Arellano, M., \& Bond, S. (1991). Some Tests of Specification for Panel Data: Monte Carlo Evidence and an Application to Employment Equations, 58(2), 277-297. Retrieved from http://people.stern.nyu.edu/wgreene/Econometrics/Arellano-Bond.pdf

Banco Mundial. (2014). Educación | Data. Retrieved September 4, 2018, from https://datos.bancomundial.org/tema/educacion

Barro, R., \& Sala i Martin, X. (2009). Crecimiento económico. Reverté.

Gonzales, A. (2017). La educación como factor de reducción de la pobreza en la región Cajamarca: 2010-2017. Universidad de Cajamarca. Universidad Nacional de Cajamarca.

INEI. (2017). Cifras de pobreza. Retrieved September 4, 2018, from https://www.inei.gob.pe/cifras-de-pobreza/

Mendoza, J. (2003). Educación y Pobreza en el Perú. Pensamiento Crítico, 2(0), 069. https://doi.org/ 10.15381/pc.v2i0.9255

MINEDU. (2017). Serie desde 2016 - ESCALE - Unidad de Estadística Educativa. Retrieved September 4, 2018, from http://escale.minedu.gob.pe/ueetendencias2016? p_auth= fLPwaAE4\&p_p_id=

TendenciasActualPortlet2016_WAR_tendencias2016portlet_INSTANCE_t6xG\&p_p_lifec ycle $=1 \&$ p_p_state $=$ normal\&p_p_mode=view\&p_p_col_id=column-

$1 \&$ p_p_col_pos $=1 \&$ p_p_col_count $=3 \&$ _TendenciasActualPort

Olivia, H. C. (2017). Relación entre el nivel educativo y la pobreza en el perú durante el periodo 2002-2012. Universidad Nacinal de Trujillo.

Pereyra, J. (2002). Una medida de eficiencia del gasto público en educación: Análisis FDH para América Latina. Revista de Estudios Económicos BCRP, (8), 237-249. Retrieved from http://www.bcrp.gob.pe/docs/Publicaciones/Revista-Estudios-Economicos/08/Estudios-

Economicos-8-9.pdf, Revista Estudios Económicos

Rodríguez, A. (2017). Crecimiento económico y capital humano : metodologíla para la simulación de una variante del Modelo de Lucas con aplicación a México. Revista Mexicana de Economía y Finanzas, 12(2), 23-47.

Romer, D. (2006). Macroeconomía avanzada. McGraw-Hill. Retrieved from https://www.marcialpons.es/ libros/macroeconomia-avanzada/9788448148096/

Wooldridge, J. M. (2002). Econometric Analysis of Cross Section and Panel Data. Retrieved from https://jrvargas.files.wordpress.com/2011/01/wooldridge_j-

_2002_econometric_analysis_of_cross_section_and_panel_data.pdf 